\title{
TOPOLOGICALLY EMBEDDED PSEUDOSPHERICAL CYLINDERS
}

\author{
EMILIO MUSSO AND LORENZO NICOLODI
}

\begin{abstract}
The class of traveling wave solutions of the sine-Gordon equation is known to be in 1-1 correspondence with the class of (necessarily singular) pseudospherical surfaces in Euclidean space with screw-motion symmetry: the pseudospherical helicoids. We explicitly describe all pseudospherical helicoids in terms of elliptic functions. This solves a problem posed by Popov 30. As an application, countably many continuous families of topologically embedded pseudospherical helicoids are constructed. A (singular) pseudospherical helicoid is proved to be either a dense subset of a region bounded by two coaxial cylinders, a topologically immersed cylinder with helical self-intersections, or a topologically embedded cylinder with helical singularities, called for short a pseudospherical twisted column. Pseudospherical twisted columns are characterized by four phenomenological invariants: the helicity $\eta \in \mathbb{Z}_{2}$, the parity $\epsilon \in \mathbb{Z}_{2}$, the wave number $\mathfrak{n} \in \mathbb{N}$, and the aspect ratio $\mathfrak{d}>0$, up to translations along the screw axis. A systematic procedure for explicitly determining all pseudospherical twisted columns from the invariants is provided.
\end{abstract}

\section{INTRODUCTION}

The study of pseudospherical surfaces, i.e., surfaces in $\mathbb{R}^{3}$ with constant Gauss curvature $K=-1$, is a classical subject in differential geometry dating back to the second half of the 19th century [1, 8. The renewed interest in the subject is principally due to the fact that pseudospherical surfaces constitute an integrable system governed by the sine-Gordon equation

$$
\phi_{s s}-\phi_{t t}=\sin \phi
$$

3, 5, 24, 28, 32, 37, 38, 39. Equation (1.1) amounts to the integrability conditions (Gauss-Codazzi equations) for the linear system of Gauss-Weingarten obeyed by the tangent frame to a pseudospherical surface with respect to curvature line coordinates. It is well-known that there is a one-to-one correspondence between local solutions $\phi$ of the sine-Gordon equation with $0<\phi<\pi$ and local pseudospherical surfaces up to rigid motion. Although the sine-Gordon equation has many global solutions defined on the whole $\mathbb{R}^{2}$, the corresponding surfaces always have singularities. In fact, according to the proof of Hilbert's theorem asserting that there is no complete immersed pseudospherical surface in $\mathbb{R}^{3}$, any smooth solution $\phi: \mathbb{R}^{2} \rightarrow \mathbb{R}$ of the sine-Gordon equation attains values that are multiples of $\pi$ [12, 29]. This implies that the map $f_{\phi}: \mathbb{R}^{2} \rightarrow \mathbb{R}^{3}$ corresponding to $\phi$ is smooth but fails to be an

2000 Mathematics Subject Classification. 53C50, 53A30.

Key words and phrases. Pseudospherical surfaces, sine-Gordon equation, traveling wave solutions, pseudospherical helicoids, topologically embedded pseudospherical cylinders.

Authors partially supported by MIUR (Italy) under the PRIN project Varietà reali e complesse: geometria, topologia e analisi armonica; and by the GNSAGA of INDAM. 
immersion at points $p$ where $\sin \phi(p)=0$, i.e., when $\phi=k \pi$, for some integer $k$. At these points the map $f_{\phi}$ has rank 1 . It then follows that there is a one-to-one correspondence between smooth solutions $\phi: \mathbb{R}^{2} \rightarrow \mathbb{R}$ of the sine-Gordon equation and smooth maps $f_{\phi}: \mathbb{R}^{2} \rightarrow \mathbb{R}^{3}$ such that (1) rank $f_{\phi} \geq 1$ everywhere; and (2) if $\operatorname{rank} f_{\phi}=2$ on an open set $U$ of $\mathbb{R}^{2}$, then $f_{\phi \mid U}$ is a pseudospherical immersion (see [27, 30, 32, 39] and the references therein).

In his 2014 monograph [30, Chapter 3], Andrey Popov posed the problem of the explicit description and computation of the (necessarily singular) pseudospherical surfaces corresponding to the stationary traveling wave solutions (single-phase solutions) of the sine-Gordon equation [1, 15, 25, 30, 32. Such surfaces are known to be the pseudospherical surfaces with screw-motion symmetry: the pseudospherical helicoids [1, 8. Examples of pseudospherical helicoids include the well-known Dini helicoids which contain the pseudosphere and correspond to the 1-soliton solutions of the sine-Gordon equation. In this paper, we explicitly determine all pseudospherical helicoids and investigate their global geometry. This leads, in particular, to the discovery of countably many continuous families of topologically embedded examples of singular pseudospherical helicoids, namely topologically embedded pseudospherical cylinders. Our study is related to recent work on pseudospherical surfaces with singularities [2, 30] and modern applications of the geometry of pseudospherical helicoids to the study of modulated wave solutions of certain mathematical models in nonlinear elasticity [33, 34, 35.

While the local geometry of pseudospherical helicoids was well understood already by the end of the 19th century [1, little is known about their global geometry (see [30 for an updated overview). It is our purpose to address natural global questions such as (1) find explicit expression for the (necessarily singular) map $f_{\phi}$ corresponding to a stationary traveling wave solution $\phi$ of the sine-Gordon equation; and (2) construct examples of singular pseudospherical helicoids which are topologically embedded cylinders, other than the pseudosphere and the rotation pseudospherical surfaces of cylindrical type. Starting from a single-phase solution $\phi$ to the sine-Gordon equation, we will explicitly compute, in terms of elliptic functions, the map $f_{\phi}$ that provides a global parametrization of the pseudospherical helicoid corresponding to $\phi$. With this in hand, we will prove the existence and construct continuous families of pseudospherical helicoids which are topologically embedded cylinders and have helical singularities. Although rather simple from a conceptual point of view, the above geometric problems present a certain computational difficulty due to the presence of the singularities and the fact that the single-phase solutions of the sine-Gordon equation are in general transcendental elliptic functions. This requires an additional detailed study of the properties of Jacobian functions which is an interesting topic on its own [30, §3.3.3]. The theoretical approach to the problem is based on the method of moving frames 9 , 10, 13.

As for constant mean curvature (CMC) surfaces, we mention that helicoidal CMC surfaces were studied by do Carmo and Dajczer [6] and further by Roussos [36] and Perdomo [26, among others. Such surfaces, and in particular helicoidal CMC cylinders, were also studied by Burstall and Kilian [4] using methods from the theory of equivariant harmonic maps and integrable systems. Notice that non-rotational helicoidal CMC surfaces are always nonsingular and have self-intersections along helices, which implies they can never be embedded. 
1.1. Description of results. We will now briefly describe our main results. A helicoid, or a helicoidal surface, is a surface $S$ in $\mathbb{R}^{3}$, possibly with singularities, swept out by an appropriately chosen planar curve $\Gamma$ animated by a helicoidal motion around an oriented line. The orbits of this motion (helices) through the initial profile curve foliate the helicoid. A helicoid $S$ is of translational kind if its profile curve $\Gamma$ is simple, has a symmetry group consisting of a nontrivial group of translations along the axis of the helicoidal motion, and has only ordinary cusps as possible singular points (cf. Figures 3 and 5). Associated with a translational helicoid there are four main phenomenological invariants: the helicity $\eta \in \mathbb{Z}_{2}$; the parity $\epsilon \in \mathbb{Z}_{2}$; the wave number $\mathfrak{n} \in \mathbb{R}^{+}$; and the aspect ratio $\mathfrak{d} \in \mathbb{R}^{+}$(cf. Section 2 for the precise definitions) 1 Depending on whether the wave number $\mathfrak{n}$ is an irrational, a rational, or a natural number, a translational helicoid $S$ is a dense subset of a region of $\mathbb{R}^{3}$ bounded by two coaxial cylinders, a topologically immersed cylinder with helical self-intersections, or a topologically embedded cylinder, that is, a closed subset of $\mathbb{R}^{3}$ homeomorphic to $S^{1} \times \mathbb{R}$. The helicoids of the last class are called twisted columns. The first main result of the paper will be the following.

Theorem 1. All pseudospherical helicoids are of translational kind.

Our second main result characterizes pseudospherical twisted columns in terms of the four phenomenological invariants and provides an effective method for explicitly constructing all of them. We will prove the following.

Theorem 2. For given $(\eta, \epsilon, \mathfrak{n}, \mathfrak{d}) \in \mathbb{Z}_{2} \times \mathbb{Z}_{2} \times \mathbb{N} \times \mathbb{R}^{+}$, there exists a unique congruence class of pseudospherical twisted columns with helicity $\eta$, parity $\epsilon$, wave number $\mathfrak{n}$, and aspect ratio $\mathfrak{d}$.

The proof of Theorem 2 is constructive and can be used to actually compute explicit parametrizations of pseudospherical twisted columns in terms of the phenomenological invariants with the help of any symbolic manipulation program supporting elliptic functions and integrals. The explicit construction also requires some numerics for the determination of the zeros of an analytic function (cf. Section 4) ${ }^{2}$

The paper is organized as follows. Section 2 recalls the one-to-one correspondence between local solutions of the sine-Gordon equation and local pseudospherical surfaces in $\mathbb{R}^{3}$ and the implicit characterization of pseudospherical helicoids in terms of stationary traveling wave solutions of the sine-Gordon equation [1, 27, 30, 33. As a consequence, it follows that the congruence classes of pseudospherical helicoids only depend on two real parameters $(\mu, r)$ and the helicity $\eta$. For $\mu=1$, we have the pseudospherical helicoids of Dini, while for $r=0$ the helicoid reduces to a surface of revolution. Being the geometry of such surfaces well known [1, 8, 30, we assume that $\mu \neq 1$ and $r \neq 0$. As a first result, we prove that two pseudospherical helicoids with parameters $(\mu, r)$ and $(\mu,-r)$, as well as with parameters $(\mu, r)$ and $(\mu /(\mu-1), r)$, are mirror images of each other (cf. Proposition 5). Thus, with no loss of generality, we may and do restrict our analysis to pseudospherical helicoids with parameters $\mu>0, \mu \neq 1$, and $r>0$.

Section 3 computes explicit global parametrizations for pseudospherical helicoids with parameters $\mu>0, \mu \neq 1$, and $r>0$. Following [30, §3.3.3], this is

\footnotetext{
${ }^{1}$ Here $\mathbb{Z}_{2}=\{ \pm 1\}$ and $\mathbb{R}^{+}$stands for the set of positive reals.

2 The proof of the theorem can also be used to find all linear Weingarten twisted columns of hyperbolic type, that is, the twisted columns such that $\alpha K+\beta H=1$, where $\beta \neq 0$ and $\alpha$ are two constants satisfying $\alpha+\beta^{2} / 4<0$ and $K$ and $H$ are the Gauss and mean curvature, respectively.
} 
achieved by dividing pseudospherical helicoids in two classes: that of magnetic-type pseudospherical helicoids, for which $\mu>1$; and that of electric-type pseudospherical helicoids, for which $\mu \in(0,1)$. For either class we compute explicit global parametrizations in terms of Jacobian elliptic functions and integrals (cf. Theorems 6 and 7). Operatively, such parametrizations are obtained by applying the method of moving frames for constructing appropriate lifts to the group of Euclidean motions. For the general theory of the method of moving frames and some applications we refer to [9, 10, 13.

Section 4 proves Theorems 1 and 2 First, we show that any pseudospherical helicoid is of translational kind. This is achieved by Lemmas 8 and 9, where we determine plane profiles for the two classes of pseudospherical helicoids and compute their phenomenological invariants in terms of the parameters $(\mu, r)$. An important consequence of Lemmas 8 and 9 is that any congruence class of pseudospherical twisted columns has a model pseudospherical twisted column. This is a typical feature of the application of the method of moving frames to geometric problems governed by integrable systems (for similar situations in other geometric contexts, see e.g. [7, 18-24]). Next, we prove the existence of pseudospherical twisted columns with prescribed invariants by showing that certain suitable modular curves intersect each other transversally at a single point (cf. Lemmas 10 and 11). Finally, we show that two pseudospherical twisted columns with different invariants cannot be congruent (cf. Lemma 12).

As a basic reference for elliptic functions and integrals we refer to [14. We warn the reader that we use the Jacobi parameter $m=k^{2}$ instead of the Jacobi elliptic modulus $k$. Symbolic and numerical computations, as well as graphics, are made with the help of the software package Mathematica 11.

\section{Pseudospherical helicoids: Definitions AND PRELIMINARY RESUlts}

In this section, after recalling the basic definitions, we prove some preliminary results about pseudospherical helicoids.

2.1. Helicoids and twisted columns. Let $\ell$ be an oriented line in $\mathbb{R}^{3}$ oriented by the unit vector $\vec{\ell}$. For $\mathfrak{p}>0$ and $\eta \in \mathbb{Z}_{2}=\{ \pm 1\}$, let $\mathrm{E}_{v}^{\ell, \mathfrak{p}, \eta}: \mathbb{R}^{3} \rightarrow \mathbb{R}^{3}$ be the 1-parameter subgroup of rigid motions of $\mathbb{R}^{3}$ given by

$$
\mathrm{E}_{v}^{\ell, \mathfrak{p}, \eta}(\mathbf{x})=\mathrm{R}_{2 \pi v}^{\ell}(\mathbf{x})+\eta v \mathfrak{p} \vec{\ell}, \quad v \in \mathbb{R},
$$

where $\mathrm{R}_{2 \pi v}^{\ell}$ denotes the oriented rotation of an angle $2 \pi v$ around $\ell$. The elements of $\mathrm{E}_{v}^{\ell, \mathfrak{p}, \eta}$ are helicoidal transformations around the screw axis $\ell$ with pitch $\mathfrak{p}$ and helicity $\eta$.

Definition 1. Let $\widetilde{\Gamma} \subset \mathbb{R}^{3}$ be a connected curve, possibly with isolated singularities. The subset $S \subset \mathbb{R}^{3}$ defined by

$$
S=\bigcup_{v \in \mathbb{R}} \mathrm{E}_{v}^{\ell, \mathfrak{p}, \eta}(\widetilde{\Gamma})
$$

is said a helicoid (or a helicoidal surface) with screw axis $\ell$, pitch $\mathfrak{p}$, helicity $\eta$, and profile $\widetilde{\Gamma}$. The profile curve $\widetilde{\Gamma}$ generating $S$ is not unique. The helicoid $S$ is right-handed or left-handed according as the helicity $\eta=1$, or $\eta=-1$. 
Definition 2. Let $S \subset \mathbb{R}^{3}$ be a helicoid and let $\mathcal{H}_{\ell}$ be one of the upper half-planes bounded by the screw axis $\ell$. The helicoid $S$ is said of translational kind if there exists a planar profile $\Gamma \subset \mathcal{H}_{\ell}$ for $S$ satisfying the following conditions:

(1) the full symmetry group of $\Gamma$ is a discrete nontrivial subgroup $\mathcal{T} \subset \mathbf{E}(3)$ of pure translations along the screw axis $\ell$ (this excludes the possibility of a rectilinear profile);

(2) the curve $\Gamma$ is a simple curve of $\mathcal{H}_{\ell}$ with only ordinary cusps as possible singular points;

(3) $\Gamma \cap \ell=\emptyset$.

If $\mathrm{T}_{\mathfrak{w} \vec{\ell}}: \mathbb{R}^{3} \rightarrow \mathbb{R}^{3}, \mathbf{x} \mapsto \mathbf{x}+\mathfrak{w} \vec{\ell}, \mathfrak{w}>0$, is the generator of the subgroup $\mathcal{T}$, we call $\mathfrak{w}$ the wavelength and $\mathfrak{n}=\mathfrak{p} / \mathfrak{w}$ the wave number of $S$.

Remark 1. If the wave number $\mathfrak{n}$ is an irrational number, then $S$ is dense in a region bounded by two coaxial cylinders. If $\mathfrak{n}$ is a rational number but not an integer, then $S$ is the image of a topological immersion of a cylinder with selfintersections along a finite number of helices. If $\mathfrak{n}$ is an integer, then $S$ is a closed subset of $\mathbb{R}^{3}$ homeomorphic to $S^{1} \times \mathbb{R}$.

Definition 3. A translational helicoid with $\mathfrak{n} \in \mathbb{N}$ is said a twisted column.

Definition 4. For a helicoid $S$ of translational type, let $\Gamma_{*}$ denote the fundamental domain of $\Gamma$ with respect to the action of the subgroup $\mathcal{T}$. Let $\mathfrak{h}$ be the number of singular points in the fundamental domain $\Gamma_{*}$. We call $\epsilon=(-1)^{\mathfrak{h}} \in \mathbb{Z}_{2}$ the parity of $S$. Let $\mathfrak{r}^{-}$and $\mathfrak{r}^{+}$be the inner and outer radii, defined respectively by

$$
\mathfrak{r}^{-}=\min _{\mathbf{x} \in \Gamma} d(\mathbf{x}, \ell), \quad \mathfrak{r}^{+}=\max _{\mathbf{x} \in \Gamma} d(\mathbf{x}, \ell) .
$$

The quotients $\mathfrak{d}^{-}=\mathfrak{w} / \mathfrak{r}^{-}$and $\mathfrak{d}^{+}=\mathfrak{w} / \mathfrak{r}^{+}$are called, respectively, the inner and outer aspect ratio.

The set $S_{*}$ of regular points of a helicoid $S$ is open and dense. We say that $S$ is pseudospherical if $S_{*}$ has constant Gaussian curvature $K=-1$.

2.2. Pseudospherical helicoids and the sine-Gordon equation. We start by recalling some well-known facts about pseudospherical surfaces of Gauss curvature $K=-1$ and in particular about pseudospherical helicoids (see e.g. Bianchi [1, Chapter XV, §§245, 250], Popov [30, Chapters 2 and 3]).

Let $f: \Omega \subset \mathbb{R}^{2} \rightarrow \mathbb{R}^{3}$ is an immersion with constant Gaussian curvature $K=-1$. Then, for every $p \in \Omega$, there exists an open neighborhood $U \subset \Omega$ of $p$ and local (curvature line) coordinates $s, t$ on $U$, so that the first and second fundamental forms of $f$ read

$$
\mathbf{g}=\cos ^{2}\left(\frac{\phi}{2}\right) d s^{2}+\sin ^{2}\left(\frac{\phi}{2}\right) d t^{2}, \quad \mathbf{h}=\cos \left(\frac{\phi}{2}\right) \sin \left(\frac{\phi}{2}\right)\left(d s^{2}-d t^{2}\right),
$$

where $\phi: U \rightarrow \mathbb{R}$ (the angular function) is a solution of the sine-Gordon partial differential equation,

$$
\phi_{s s}-\phi_{t t}=\sin \phi
$$

satisfying $\phi(U) \subset(0, \pi)$.

Conversely, if $\Omega \subset \mathbb{R}^{2}$ is simply connected and $\phi=\phi(s, t): \Omega \rightarrow \mathbb{R}$ is a nonconstant solution of the sine-Gordon equation 2.1 such that $\phi(\Omega) \subset(0, \pi)$, then 
there exists an immersion $f: \Omega \rightarrow \mathbb{R}^{3}$ of constant Gaussian curvature $K=-1$ with fundamental forms

$$
\mathbf{g}_{\phi}=\cos ^{2}\left(\frac{\phi}{2}\right) d s^{2}+\sin ^{2}\left(\frac{\phi}{2}\right) d t^{2}, \quad \mathbf{h}_{\phi}=\cos \left(\frac{\phi}{2}\right) \sin \left(\frac{\phi}{2}\right)\left(d s^{2}-d t^{2}\right) .
$$

The immersion $f$ is implicitly defined by $\mathbf{g}_{f}$ and $\mathbf{h}_{f}$ and is unique up to rigid motions of $\mathbb{R}^{3}$.

This latter result have been generalized by Poznyak [31] (see also [30, Theorem 2.7.1] and [27, p. 55]) to the case where the solution $\phi$ is not subject to the condition that its image is contained in $(0, \pi)$ and has low differentiability.

Theorem 3. Let $\phi=\phi(s, t): \mathbb{R}^{2} \rightarrow \mathbb{R}$ be a nonconstant $C^{4}$-solution of the sineGordon equation (2.1). Then there exists a $C^{2}$-map $f_{\phi}: \mathbb{R}^{2} \rightarrow \mathbb{R}^{3}$ whose restriction to the open set $\left\{p \in \mathbb{R}^{2}: \phi(p) \neq k \pi, k \in \mathbb{Z}\right\}$ is a pseudospherical immersion with first and second fundamental forms given by

$$
\mathbf{g}_{\phi}=\cos ^{2}\left(\frac{\phi}{2}\right) d s^{2}+\sin ^{2}\left(\frac{\phi}{2}\right) d t^{2} \quad \text { and } \quad \mathbf{h}_{\phi}=\cos \left(\frac{\phi}{2}\right) \sin \left(\frac{\phi}{2}\right)\left(d s^{2}-d t^{2}\right) .
$$

Remark 2. The map $f_{\phi}: \mathbb{R}^{2} \rightarrow \mathbb{R}^{3}$ corresponding to a smooth solution $\phi: \mathbb{R}^{2} \rightarrow \mathbb{R}$ of the sine-Gordon equation (2.1) is smooth but fails to be an immersion at points $p$ where $\phi(p)=k \pi$, for some integer $k$. At these points the map $f_{\phi}$ has rank 1. It follows that there is a one-to-one correspondence between smooth solutions $\phi: \mathbb{R}^{2} \rightarrow \mathbb{R}$ of the sine-Gordon equation and smooth maps $f_{\phi}: \mathbb{R}^{2} \rightarrow \mathbb{R}^{3}$ such that (1) $\operatorname{rank} f_{\phi} \geq 1$ everywhere; and (2) if $\operatorname{rank} f_{\phi}=2$ on an open set $U$ of $\mathbb{R}^{2}$, then $f_{\phi \mid U}$ is a pseudospherical immersion (see e.g. [27, 30, 32, 39]).

In particular, we have the following (see 30]).

Corollary 4. The map $f_{\phi}$ parametrizes a pseudospherical helicoid or a pseudospherical surface of revolution if and only if the angular function $\phi$ is a stationary traveling wave solution of (2.1), that is, a solution of the form

$$
\phi=\phi(s, t)=\lambda(\xi), \quad \xi=a s+b t,
$$

where $\lambda$ is a function of one variable and $a, b$ are real constants with $a \neq 0, a^{2} \neq b^{2}$. We call $\phi$ the potential of the map $f_{\phi}$.

Remark 3. The map $f_{\phi}$ exists globally. However, its explicit expression seems not to have been computed in the literature (see [30]).

If we let $a=\cosh r / L$ and $b=\sinh r / L$, the function $\lambda$ satisfies the simple pendulum equation $\lambda^{\prime \prime}-L^{2} \sin \lambda=0$. Consequently, a stationary traveling wave solution of the sine-Gordon equation depend on two real parameters $\mu$ and $r$. For given $\mu$ and $r$, the corresponding traveling wave solution $\phi_{\mu, r}$ of the sine-Gordon equation can be written, up to an affine change of variables, as

$$
\begin{aligned}
& \phi_{\mu, r}(s, t)=-2 \operatorname{am}\left(\frac{\cosh r}{\sqrt{|\mu|}} s+\frac{\sinh r}{\sqrt{|\mu|}} t, \mu\right), \quad \mu<0 \\
& \phi_{1, r}(s, t)=-4 \arctan \left(e^{r s+\sqrt{1+r^{2}} t}\right)+\pi, \\
& \phi_{\mu, r}(s, t)=-2 \operatorname{am}\left(\frac{\sinh r}{\sqrt{|\mu|}} s+\frac{\cosh r}{\sqrt{|\mu|}} t, \mu\right), \quad \mu>0, \mu \neq 1,
\end{aligned}
$$

where $\operatorname{am}(-, \mu)$ is the Jacobian amplitude with parameter $\mu$. 
Remark 4 (Jacobian elliptic functions and integrals [14). To fix notation, we recall some basic definitions about the Jacobian elliptic functions and integrals. If

$$
u=F(\varphi, \mu)=\int_{0}^{\varphi} \frac{d \theta}{\left(1-\mu \sin ^{2} \theta\right)^{\frac{1}{2}}},
$$

then $\operatorname{am}(u, \mu)=F^{-1}(u, \mu)=\varphi$ is the Jacobian amplitude with parameter $\mu$. The Jacobian elliptic functions $\operatorname{sn}(-, \mu), \operatorname{cn}(-, \mu)$ and $\operatorname{dn}(-, \mu)$ with parameter $\mu$ are defined by

$$
\operatorname{sn}(u, \mu)=\sin \varphi, \quad \operatorname{cn}(u, \mu)=\cos \varphi, \quad \operatorname{dn}(u, \mu)=\left(1-\mu \sin ^{2} \varphi\right)^{\frac{1}{2}} .
$$

The integral $F(\varphi, \mu)$ given by the formula above is called the incomplete elliptic integral of the first kind. The complete elliptic integral of the first kind is defined by $K(\mu)=F\left(\frac{\pi}{2}, \mu\right)$. The integral defined by

$$
E(\varphi, \mu)=\int_{0}^{\varphi}\left(1-\mu \sin ^{2} \theta\right)^{\frac{1}{2}} d \theta
$$

is called the incomplete elliptic integral of the second kind. The complete elliptic integral of the second kind is defined by $E(\mu)=E\left(\frac{\pi}{2}, \mu\right)$. The integral defined by

$$
\Pi(n, \varphi, \mu)=\int_{0}^{\varphi} \frac{d \theta}{\left(1-n \sin ^{2} \theta\right)\left(1-\mu \sin ^{2} \theta\right)^{\frac{1}{2}}}
$$

is called the incomplete elliptic integral of the third kind. The complete elliptic integral of the third kind is defined by $\Pi(n, \mu)=\Pi\left(n, \frac{\pi}{2}, \mu\right)$.

In the following, we will denote by $S_{\mu, r}$ the pseudospherical helicoid associated with the potential $\phi_{\mu, r}$ and parametrized by the map $f_{\mu, r}$. We will briefly refer to $S_{\mu, r}$ as the pseudospherical helicoid with parameters $(\mu, r)$.

Remark 5. If $r=0$, the pseudospherical helicoid reduces to a pseudospherical surface of revolution, while if $\mu=1$ we obtain the well-known pseudospherical helicoids of Dini 1, 11, 15. If $\mu=1$ and $r=0$, we obtain the Beltrami pseudosphere [1, 8. In the following, we will assume that $\mu \neq 1$ and $r \neq 0$.

Let $S_{\mu, r}$ be a pseudospherical helicoid with parameters $(\mu, r)$ and let $\Phi_{\mu, r}$ be the linear change of variables $(s, t)=\Phi_{\mu, r}(u, v)$, where

$$
\Phi_{\mu, r}(u, v)=(-\sqrt{|\mu|} \sinh (r) u+\sqrt{|\mu|} \cosh (r) v, \sqrt{|\mu|} \cosh (r) u-\sqrt{|\mu|} \sinh (r) v) .
$$

It follows that the fundamental forms of the reparametrization $\mathrm{L}_{\mu, r}=f_{\mu, r} \circ \Phi_{\mu, r}$ are

$$
\begin{gathered}
\mathbf{g}_{\mu, r}=|\mu|\left(\operatorname{sn}^{2}(u, \mu)+\sinh ^{2}(r)\right) d u^{2}-\frac{|\mu| \sinh (2 r)}{2} d u d v \\
+|\mu|\left(\operatorname{cn}^{2}(u, \mu)+\sinh ^{2}(r)\right) d v^{2}, \\
\mathbf{h}_{\mu, r}=\mu \operatorname{sn}(u, \mu) \operatorname{cn}(u, \mu)\left(d u^{2}-d v^{2}\right) .
\end{gathered}
$$

We can now prove the following.

Proposition 5. (1) A pseudospherical helicoid $S_{\mu, r}$ with parameters $(\mu, r), \mu<0$, and a pseudospherical helicoid $S_{\mu^{\prime}, r}$ with parameters $\left(\mu^{\prime}, r\right), \mu^{\prime}=\mu /(\mu-1) \in$ $(0,1)$, are mirror images of each other. (2) A pseudospherical helicoid $S_{\mu, r}$ with parameters $(\mu, r)$ and a pseudospherical helicoid $S_{\mu,-r}$ with parameters $(\mu,-r)$ are mirror images of each other. 
Proof. (1) Let $K(\mu)$ be the complete elliptic integral of the first kind with parameter $\mu$. Using the basic modular transformations of the Jacobian elliptic functions (cf. [14, p. 38 and p. 77]), we obtain

$$
\begin{aligned}
& \operatorname{cn}(u, \mu)=\operatorname{sn}\left(\sqrt{1-\mu} u+K\left(\mu^{\prime}\right), \mu^{\prime}\right), \\
& \operatorname{sn}(u, \mu)=-\operatorname{cn}\left(\sqrt{1-\mu} u+K\left(\mu^{\prime}\right), \mu^{\prime}\right),
\end{aligned}
$$

and

$$
K\left(\mu^{\prime}\right)=\sqrt{1-\mu} K(\mu) .
$$

Consider the linear change of variables

$$
\Phi^{\prime}:(u, v) \mapsto \frac{1}{\sqrt{1-\mu}}\left(K\left(\mu^{\prime}\right)-v,-u\right) .
$$

Then, using (2.3) and (2.4), we deduce that the fundamental forms of $-\mathrm{L}_{\mu, r} \circ \Phi^{\prime}$ coincide with those of $\mathrm{L}_{\mu^{\prime}, r}$.

(2) We conclude the proof observing that the fundamental forms of $-\mathrm{L}_{\mu, r}(u,-v)$ coincide with those of $\mathrm{L}_{\mu,-r}$.

Remark 6. Henceforth, without loss of generality, we shall restrict to pseudospherical helicoids with parameters $\mu>0, \mu \neq 1$, and $r>0$.

\section{EXPLiCIT INTEGRATION OF PSEUDOSPHERICAL HELICOIDS}

In this section we will find explicit parametrizations of pseudospherical helicoids in terms of elliptic functions and elliptic integrals. To this end, following Popov [30. Chapter 3], pseudospherical helicoids are divided in two classes.

Definition 5. A pseudospherical helicoid with parameters $(\mu, r)$ is said of magnetic type (resp., electric type) if $\mu>1$ (resp., $\mu \in(0,1)$ ).

3.1. Pseudospherical helicoids of magnetic type. For $\mu>1$ and $r>0$, let

$$
\begin{aligned}
m:=\mu^{-1} \in & (0,1), \\
\psi_{\mu, r}(u)= & \sqrt{m+\sinh ^{2}(r)} \operatorname{coth}(r) \Pi\left(-m \operatorname{csch}^{2}(r), m\right) \\
& -\sqrt{m+\sinh ^{2}(r)} \operatorname{coth}(r) \Pi\left(-m \operatorname{csch}^{2}(r), \operatorname{am}(u, m), m\right), \\
\xi_{\mu, r}(u)= & \operatorname{dn}(u, m) \operatorname{sn}^{2}(u, m) \sqrt{m \operatorname{sn}^{2}(u, m)+\sinh ^{2}(r),} \\
\zeta_{\mu, r}(u)= & \frac{1}{\sqrt{m+\sinh ^{2}(r)}}\left(E(m)+\cosh ^{2}(r)(u-K(m))\right. \\
& -E(\operatorname{am}(u, m), m)),
\end{aligned}
$$

and

$$
\begin{aligned}
q_{\mu, r}^{1}(u) & =\frac{-\sqrt{m}}{\sqrt{m+\sinh ^{2}(r)} \operatorname{sn}(u, m)\left(m \operatorname{sn}^{2}(u, m)+\sinh ^{2}(r)\right)}, \\
q_{\mu, r}^{2}(u) & =\frac{\sqrt{m} \cosh (r) \sinh (r) \operatorname{cn}(u, m)}{\left(m+\sinh ^{2}(r)\right) \operatorname{sn}^{2}(u, m) \operatorname{dn}(u, m)\left(m \operatorname{sn}^{2}(u, m)+\sinh ^{2}(r)\right)}, \\
\rho_{\mu, r} & =-2 \pi \frac{\cosh (r) \sinh ^{2}(r)}{m+\sinh ^{2}(r)} .
\end{aligned}
$$


Let $\widetilde{\Gamma}_{\mu, r} \subset \mathbb{R}^{3}$ be the trajectory of the parametrized curve

$$
\widetilde{\gamma}_{\mu, r}=\left(\widetilde{x}_{\mu, r}, \widetilde{y}_{\mu, r}, \widetilde{z}_{\mu, r}\right): \mathbb{R} \rightarrow \mathbb{R}^{3}
$$

defined by

$$
\begin{aligned}
\widetilde{x}_{\mu, r} & =\xi_{\mu, r}\left(\cos \left(\psi_{\mu, r}\right) q_{\mu, r}^{1}-\sin \left(\psi_{\mu, r}\right) q_{\mu, r}^{2}\right) \\
\widetilde{y}_{\mu, r} & =\xi_{\mu, r}\left(\sin \left(\psi_{\mu, r}\right) q_{\mu, r}^{1}+\cos \left(\psi_{\mu, r}\right) q_{\mu, r}^{2}\right) \\
\widetilde{z}_{\mu, r} & =\zeta_{\mu, r} .
\end{aligned}
$$

We can now state the following.

Theorem 6. Let $S_{\mu, r}$ be a pseudospherical helicoid of magnetic type, that is, a pseudospherical helicoid with parameters $\mu>1$ and $r>0$. Then, the real analytic map $\widetilde{f}_{\mu, r}=\left(\widetilde{f}_{\mu, r}^{1}, \widetilde{f}_{\mu, r}^{2}, \widetilde{f}_{\mu, r}^{3}\right): \mathbb{R}^{2} \rightarrow \mathbb{R}^{3}$ with components

$$
\begin{aligned}
& \widetilde{f}_{\mu, r}^{1}(u, v)=\cos (2 \pi v) \widetilde{x}_{\mu, r}(u)-\sin (2 \pi v) \widetilde{y}_{\mu, r}(u), \\
& \widetilde{f}_{\mu, r}^{2}(u, v)=\sin (2 \pi v) \widetilde{x}_{\mu, r}(u)+\cos (2 \pi v) \widetilde{y}_{\mu, r}(u), \\
& \widetilde{f}_{\mu, r}^{3}(u, v)=\widetilde{z}_{\mu, r}(u)+\rho_{\mu, r} v
\end{aligned}
$$

is a global parametrization of $-S_{\mu, r}{ }^{3}$

Proof. Using any software of symbolic computation implementing elliptic functions such as Mathematica, one can see that the coefficients of the fundamental forms of $\widetilde{f}_{\mu, r}$ are

$$
\begin{aligned}
& \widetilde{g}_{11}^{\mu, r}=m \operatorname{sn}^{2}(u, m)+\sinh ^{2}(r) \\
& \widetilde{g}_{22}^{\mu, r}=\frac{4 \pi^{2}\left(1+\cosh (2 r)-2 m \operatorname{sn}^{2}(u, m)\right)}{-1+2 m+\cosh (2 r)} \\
& \widetilde{g}_{12}^{\mu, r}=\frac{-\sqrt{2} \pi \sinh (2 r)}{\sqrt{-1+2 m+\cosh (2 r)}} \\
& \widetilde{h}_{11}^{\mu, r}=-\sqrt{m} \operatorname{dn}(u, m) \operatorname{sn}(u, m) \\
& \widetilde{h}_{22}^{\mu, r}=\frac{8 \pi^{2} \sqrt{m} \operatorname{dn}(u, m) \operatorname{sn}(u, m)}{-1+2 m+\cosh (2 r)} \\
& \widetilde{h}_{12}^{\mu, r}=0 .
\end{aligned}
$$

Using the parameter transformations of Jacobian functions (see [14, p. 77])

$$
\operatorname{sn}(u, h)=\frac{1}{\sqrt{h}} \operatorname{sn}\left(u \sqrt{h}, h^{-1}\right), \quad \operatorname{cn}(u, h)=\operatorname{dn}\left(u \sqrt{h}, h^{-1}\right)
$$

\footnotetext{
${ }^{3}$ Here $-S_{\mu, r}$ stands for $-\mathrm{id}_{\mathbb{R}^{3}}\left(S_{\mu, r}\right)$, where $\mathrm{id}_{\mathbb{R}^{3}}$ denotes the identity map of $\mathbb{R}^{3}$.
} 
and taking into account the expressions 2.2 , the coefficients $g_{i j}^{\mu, r}$ and $h_{i j}^{\mu, r}$ of the fundamental forms of $\mathrm{L}_{\mu, r}$ can be written as

$$
\begin{aligned}
& g_{11}^{\mu, r}=\operatorname{sn}^{2}(u / \sqrt{m}, m)+\frac{1}{m} \sinh ^{2}(r), \\
& g_{22}^{\mu, r}=\frac{1}{m}\left(\operatorname{dn}^{2}(u / \sqrt{m}, m)+\sinh ^{2}(r)\right), \\
& g_{12}^{\mu, r}=-\frac{1}{m} \sinh (r) \cosh (r), \\
& h_{11}^{\mu, r}=-h_{22}^{\mu, r}=\frac{1}{\sqrt{m}} \operatorname{dn}(u / \sqrt{m}, m) \operatorname{sn}(u / \sqrt{m}, m), \\
& h_{12}^{\mu, r}=0 .
\end{aligned}
$$

Next, consider the linear change of variables

$$
\Phi^{\prime \prime}: \mathbb{R}^{2} \ni(u, v) \mapsto \sqrt{m}\left(u, \frac{2 \sqrt{2} \pi}{\sqrt{2 m-1+\cosh (2 r)}} v\right) \in \mathbb{R}^{2} .
$$

From 3.5 it follows that the first quadratic form of the reparametrization $L_{\mu, r} \circ \Phi^{\prime \prime}$ coincide with the first quadratic form of $\widetilde{f}_{\mu, r}$ and that the second quadratic form of $L_{\mu, r} \circ \Phi^{\prime \prime}$ is the opposite of the second quadratic form of $\tilde{f}_{\mu, r}$. This proves the result.

3.2. Pseudospherical helicoids of electric type. For given $\mu \in(0,1)$ and $r>0$, let

$$
\begin{aligned}
& m:=\mu^{-1}>1, \\
& \psi_{\mu, r}(u)=-\frac{\sqrt{m+\sinh ^{2}(r)} \operatorname{coth}(r)}{\sqrt{m}} \Pi\left(-\operatorname{csch}^{2}(r), \operatorname{am}\left(\sqrt{m} u, \frac{1}{m}\right), \frac{1}{m}\right), \\
& \zeta_{\mu, r}(u)=\frac{\left(\cosh ^{2}(r)+m-1\right) u-\sqrt{m} E\left(\operatorname{am}\left(\sqrt{m} u, \frac{1}{m}\right), \frac{1}{m}\right)}{\sqrt{m+\sinh ^{2}(r)}},
\end{aligned}
$$

Note that $\psi_{\mu, r}$ is quasi-periodic, with quasi-period $2 K(1 / m) / \sqrt{m}$. Next, define the real-analytic functions

$$
\begin{aligned}
\xi_{\mu, r}(u) & =\sqrt{\frac{m}{m \operatorname{sn}^{2}(u, m)+\sinh ^{2}(r)}}, \\
q_{\mu, r}^{1}(u) & =\frac{\cosh (r) \sinh (r)}{m+\sinh ^{2}(r)} \operatorname{cn}(u, m), \\
q_{\mu, r}^{2}(u) & =\frac{\operatorname{dn}(u, m) \operatorname{sn}(u, m)}{\sqrt{m+\sinh ^{2}(r)}}
\end{aligned}
$$

and the constant

$$
\rho_{\mu, r}=-2 \pi \frac{\cosh (r) \sinh (r)}{m+\sinh ^{2}(r)}
$$

Let $\widetilde{\Gamma}_{\mu, r} \subset \mathbb{R}^{3}$ be the trajectory of the parametrized curve

$$
\widetilde{\gamma}_{\mu, r}=\left(\widetilde{x}_{\mu, r}, \widetilde{y}_{\mu, r}, \widetilde{z}_{\mu, r}\right): \mathbb{R} \rightarrow \mathbb{R}^{3}
$$


defined by

$$
\begin{aligned}
\widetilde{x}_{\mu, r} & =\xi_{\mu, r}\left(\cos \left(\psi_{\mu, r}\right) q_{\mu, r}^{1}-\sin \left(\psi_{\mu, r}\right) q_{\mu, r}^{2}\right), \\
\widetilde{y}_{\mu, r} & =\xi_{\mu, r}\left(\sin \left(\psi_{\mu, r}\right) q_{\mu, r}^{1}+\cos \left(\psi_{\mu, r}\right) q_{\mu, r}^{2}\right), \\
\widetilde{z}_{\mu, r} & =\zeta_{\mu, r} .
\end{aligned}
$$

We are in a position to prove the following.

Theorem 7. Let $S_{\mu, r}$ be a pseudospherical helicoid of electric type, that is, a pseudospherical helicoid with parameters $\mu \in(0,1)$ and $r>0$. Then, the real-analytic map $\widetilde{f}_{\mu, r}=\left(\widetilde{f}_{\mu, r}^{1}, \widetilde{f}_{\mu, r}^{2}, \widetilde{f}_{\mu, r}^{3}\right): \mathbb{R}^{2} \rightarrow \mathbb{R}^{3}$ with components

$$
\begin{aligned}
\widetilde{f}_{\mu, r}^{1}(u, v) & =\cos (2 \pi v) \widetilde{x}_{\mu, r}(u)-\sin (2 \pi v) \widetilde{y}_{\mu, r}(u), \\
\widetilde{f}_{\mu, r}^{2}(u, v) & =\sin (2 \pi v) \widetilde{x}_{\mu, r}(u)+\cos (2 \pi v) \widetilde{y}_{\mu, r}(u), \\
\widetilde{f}_{\mu, r}^{3}(u, v) & =\widetilde{z}_{\mu, r}(u)+\rho_{\mu, r} v
\end{aligned}
$$

is a global parametrization of $-S_{\mu, r}$.

Proof. Since the fundamental forms of $\tilde{f}_{\mu, r}$ are as in 3.4 , we can argue as in the proof of Theorem 6 .

Remark 7. Observe, however, that the profile curves computed in the two theorems above are not planar (see Figure 1).
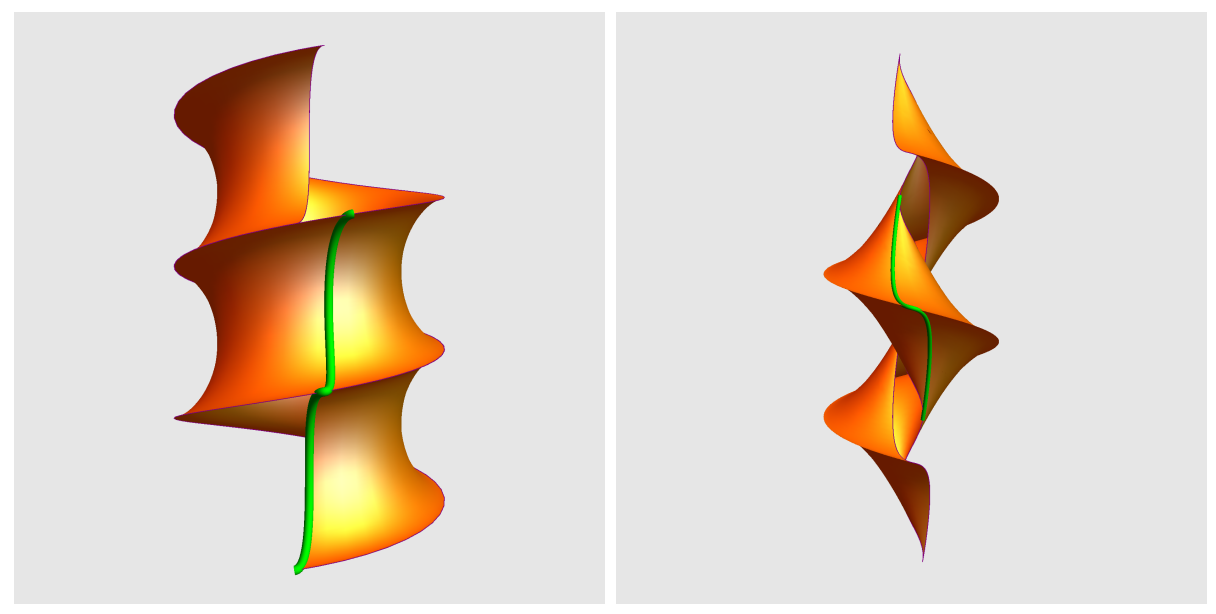

Figure 1. The fundamental domains of pseudospherical twisted columns of magnetic (on the left) and electric (on the right) types and their non-planar profiles.

\section{The PRoOF OF the MAIN THEOREMS}

This section proves Theorems 1 and 2 . The proof is divided into five lemmas. Lemmas 8 and 9 (cf. Section 4.1) prove that pseudospherical helicoids of magnetic and electric type are of translational kind (hence Theorem 1) by determining in both cases the planar profiles of the pseudospherical helicoids and by computing 
the corresponding phenomenological invariants in terms of the parameters. Lemmas 10 and 11 (cf. Section 4.2) prove the existence of pseudospherical twisted columns of magnetic and electric type with prescribed wave number and aspect ratio. Finally, Lemma 12 (cf. Section 4.3 proves that pseudospherical twisted columns with different invariants cannot be congruent.

4.1. Planar profiles and geometric invariants of pseudospherical helicoids. We start our analysis with pseudospherical helicoids of magnetic type.

Lemma 8. Any pseudospherical helicoid of magnetic type with parameters $\mu>1$ and $r>0$ is of translational kind. Moreover, it has parity $\epsilon=-1$ and its pitch, wavelength and aspect ratio are given, respectively, by

$$
\begin{aligned}
\mathfrak{p}_{\mu, r} & =2 \pi \frac{\cosh (r) \sinh (r)}{m+\sinh ^{2}(r)}, \\
\mathfrak{w}_{\mu, r} & =2 \frac{\cosh ^{2}(r) \Pi\left(-\sinh ^{2}(r), m\right)-E(m)}{\sqrt{m+\sinh ^{2}(r)}}, \\
\mathfrak{d}_{\mu, r} & =\frac{2 \sqrt{m+\sinh ^{2}(r)}\left(\cosh ^{2}(r) \Pi\left(-\sinh ^{2} r, m\right)-E(m)\right)}{\sqrt{m(1-m)}},
\end{aligned}
$$

where $m=\mu^{-1} \in(0,1)$

Proof. The profile $\widehat{\Gamma}_{\mu, r}$, although not a plane curve, does not intersect the screw axis. Therefore, for each $u \in \mathbb{R}$, we may consider the oriented upper half-plane $\mathcal{H}_{\mu, r}(u)$ bounded by the screw axis and passing through the point $\widetilde{\gamma}_{\mu, r}(u)$. Let $2 \pi \theta_{\mu, r}: \mathbb{R} \rightarrow \mathbb{R}$ be an analytic determination of the amplitude of the angle between the oriented upper half-plane $\left\{(x, y, z) \in \mathbb{R}^{3} \mid y=0, x>0\right\}$ and the oriented halfplane $\mathcal{H}_{\mu, r}(u)$. Denote by $[a]$ the integer part of a real number and by $\delta_{-}: \mathbb{R} \rightarrow \mathbb{R}$ the unit step function

$$
\begin{aligned}
& \delta_{-}(u)=1, \quad u \geq 0, \\
& \delta_{-}(u)=0, \quad u<0 .
\end{aligned}
$$

We can take as $\theta_{\mu, r}$ the unique smooth function defined on the whole real line, such that, for each $u \in \mathbb{R} \backslash\{2 h K(m), h \in \mathbb{Z}\}$,

$$
\theta_{\mu, r}(u)=\frac{1}{2 \pi}\left(\psi_{\mu, r}(u)+\arctan \left(\frac{q_{\mu, r}^{2}(u)}{q_{\mu, r}^{1}(u)}\right)+\pi\left([u / 2 K(m)]+\delta_{-}(u)\right)\right) .
$$

Consequently, the plane profile of the helicoid is the real-analytic curve $\Gamma_{\mu, r}$ in the upper half-plane $\left\{(x, y, z) \in \mathbb{R}^{3} \mid y=0, x>0\right\}$, parametrized by the map

$$
\gamma_{\mu, r}=\left(q_{\mu, r}, 0, \zeta_{\mu, r}-\rho_{\mu, r} \theta_{\mu, r}\right)
$$

where

$$
q_{\mu, r}=\xi_{\mu, r} \sqrt{\left(q_{\mu, r}^{1}\right)^{2}+\left(q_{\mu, r}^{2}\right)^{2}} .
$$

Correspondingly, we obtain the following parametrization of the helicoid in terms of the plane profile $\Gamma_{\mu, r}$,

$$
f_{\mu, r}(u, v)=\left(q_{\mu, r}(u) \cos (2 \pi v), q_{\mu, r}(u) \sin (2 \pi v), \zeta_{\mu, r}(u)-\rho_{\mu, r} \theta_{\mu, r}(u)+\rho_{\mu, r} v\right) .
$$




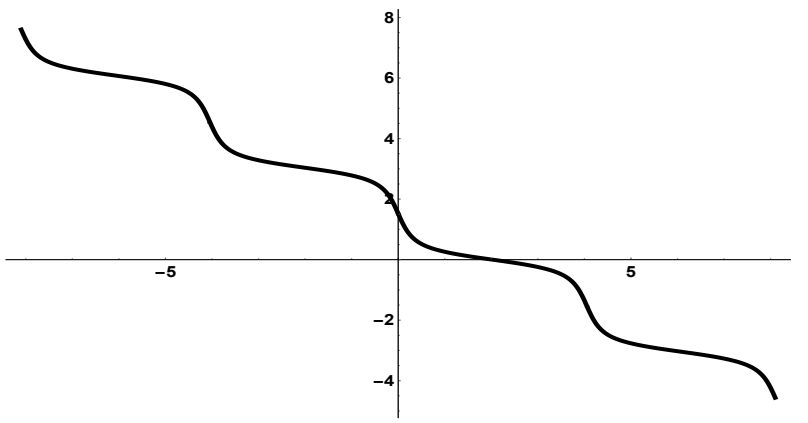

FIGURE 2. The graph of the angular function $\theta_{\mu, r}, \mu \approx 1.50742$ and $r \approx 0.208$.

It then follows that the pitch of the helicoid is

$$
\mathfrak{p}_{\mu, r}=-\rho_{\mu, r}=2 \pi \frac{\cosh (r) \sinh (r)}{m+\sinh ^{2}(r)} .
$$

The first component of $\gamma_{\mu, r}$ is periodic with minimal period $\omega_{\mu}=2 K(m)$ and the

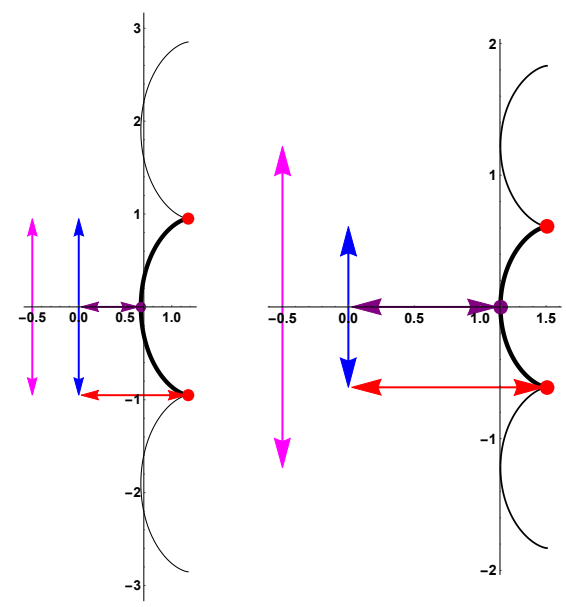

Figure 3. The planar profiles of two twisted columns of magnetic type with wave numbers 1 and 2 , respectively.

third component is a strictly decreasing quasi-periodic function with quasi-period $\omega_{\mu}$. Consequently, $\Gamma_{\mu, r}$ is invariant under the translational group along the $z$-axis and its wavelength is

$$
\mathfrak{w}_{\mu, r}=d\left[\gamma_{\mu, r}(2 K(m)), \gamma_{\mu, r}(0)\right]=2 \frac{\cosh ^{2}(r) \Pi\left(-\sinh ^{2}(r), m\right)-E(m)}{\sqrt{m+\sinh ^{2}(r)}} .
$$


The profile has countably many cusps of order 1 (ordinary cusps) located at the points

$$
\gamma_{\mu, r}(2 n K(m))=\gamma_{\mu, r}(0)+n \mathfrak{w}_{\mu, r} \vec{k}, \quad n \in \mathbb{Z},
$$

where $\vec{k}$ denotes the unit $z$-coordinate vector. All the other points are regular. This implies that the helicoid is of translational type with parity $\epsilon=-1$. The profile reaches the minimum distance from the screw axis at the points

$$
\gamma_{\mu}(K(m)+2 n K(m))=\gamma_{\mu, r}(K(m))+n \mathfrak{w}_{\mu, r} \vec{k} .
$$

The inner radius is thus

$$
\mathfrak{r}_{\mu, r}^{-}=\sqrt{\frac{m(1-m)}{m+\sinh ^{2}(r)}} .
$$

This implies that the aspect ratid ${ }^{4}$ is

$$
\mathfrak{d}_{\mu, r}=\frac{2 \sqrt{m+\sinh ^{2}(r)}\left(\cosh ^{2}(r) \Pi\left(-\sinh ^{2}(r), m\right)-E(m)\right)}{\sqrt{m(1-m)}},
$$

which completes the proof.

As for the pseudospherical helicoids of electric type, we have the following.

Lemma 9. A pseudospherical helicoid of electric type with parameters $\mu \in(0,1)$ and $r>0$ is of translational kind. Moreover, It has parity $\epsilon=1$ and its pitch, wavelength and aspect ratio are given, respectively, by

$$
\begin{aligned}
\mathfrak{p}_{\mu, r}= & 2 \pi \frac{\cosh (r) \sinh (r)}{m+\sinh ^{2}(r)}, \\
\mathfrak{w}_{\mu, r}= & 2 \frac{\left(m+\cosh ^{2}(r)-1\right) K\left(\frac{1}{m}\right)-m E\left(\frac{1}{m}\right)-\cosh ^{2}(r) \Pi\left(-\operatorname{csch}^{2}(r), \frac{1}{m}\right)}{\sqrt{m\left(m+\sinh ^{2}(r)\right)}} \\
\mathfrak{d}_{\mu, r}= & \frac{2 \sqrt{m+\sinh ^{2}(r)}}{m \cosh (r)}\left(\left(m+\cosh ^{2}(r)-1\right) K\left(\frac{1}{m}\right)-m E\left(\frac{1}{m}\right)\right. \\
& \left.-\cosh ^{2}(r) \Pi\left(-\operatorname{csch}^{2}(r), \frac{1}{m}\right)\right) \\
\text { where } m= & \mu^{-1}>1
\end{aligned}
$$

Proof. The reasoning is quite similar to the one in the previous lemma. The profile $\widetilde{\Gamma}_{\mu, r}$ does not intersect the screw axis and the function

$$
2 \pi \theta_{\mu, r}(u)=\left(\psi_{\mu, r}(u)+\arctan \left(\frac{q_{\mu, r}^{2}(u)}{q_{\mu, r}^{1}(u)}\right)\right)
$$

is an analytic determination of the amplitude of the angle formed by the upper half-plane $\left\{(x, y, z) \in \mathbb{R}^{3} \mid y=0, x>0\right\}$ and the upper half-plane bounded by the screw axis and passing through the point $\widetilde{\gamma}_{\mu, r}(u)$. Therefore, the plane profile is the real-analytic curve $\Gamma_{\mu, r}$ in the upper half-plane $\left\{(x, y, z) \in \mathbb{R}^{3} \mid y=0, x>0\right\}$, parametrized by

$$
\gamma_{\mu, r}=\left(q_{\mu, r}, 0, \zeta_{\mu, r}-\rho_{\mu, r} \theta_{\mu, r}\right),
$$

\footnotetext{
${ }^{4}$ Since $\epsilon=-1$, the aspect ratio is $\mathfrak{w} / \mathfrak{r}^{-}$.
} 
where

$$
q_{\mu, r}=\xi_{\mu, r} \sqrt{\left(q_{\mu, r}^{1}\right)^{2}+\left(q_{\mu, r}^{2}\right)^{2}} .
$$

Therefore, the parametrization of the helicoid in terms of the plane profile is

$$
f_{\mu, r}(u, v)=\left(q_{\mu, r}(u) \cos (2 \pi v), q_{\mu, r}(u) \sin (2 \pi v), \zeta_{\mu, r}(u)-\rho_{\mu, r} \theta_{\mu, r}(u)+\rho_{\mu, r} v\right) .
$$

From this it follows that the pitch of the helicoid is

$$
\mathfrak{p}_{\mu, r}=-\rho_{\mu, r}=2 \pi \frac{\cosh (r) \sinh (r)}{m+\sinh ^{2}(r)} .
$$

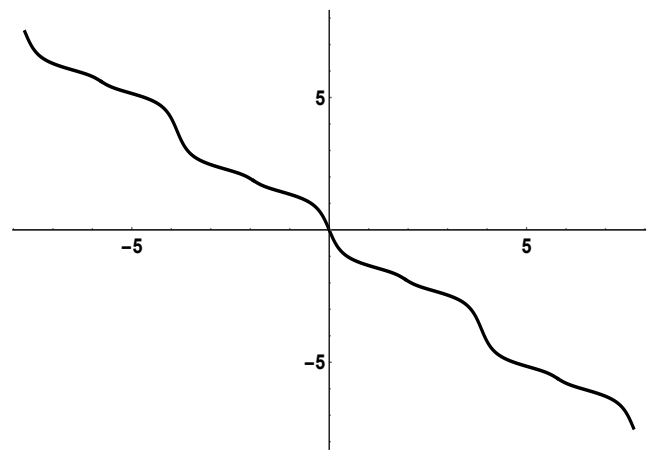

FIGURE 4. The graph of the angular function $\theta_{\mu, r}, \mu \approx 0.7707$ and $r \approx 0.20892$.

The first component of $\gamma_{\mu, r}$ is periodic with minimal period $\omega_{\mu}=2 K(1 / m) / \sqrt{m}$ while the third component is a strictly decreasing quasi-periodic function with quasi-period $\omega_{\mu}$. Consequently, $\Gamma_{\mu, r}$ is invariant by the translational group along the $z$-axis and its wavelength is

$$
\begin{aligned}
\mathfrak{w}_{\mu, r} & =d\left[\gamma_{\mu, r}\left(\omega_{\mu}\right), \gamma_{\mu, r}(0)\right] \\
& =2 \frac{\left(m+\cosh ^{2}(r)-1\right) K\left(\frac{1}{m}\right)-m E\left(\frac{1}{m}\right)-\cosh ^{2}(r) \Pi\left(-\operatorname{csch}^{2}(r), \frac{1}{m}\right)}{\sqrt{m\left(m+\sinh ^{2}(r)\right)}} .
\end{aligned}
$$

The profile possesses countably many cusps of order 1 (ordinary cusps) located at the points

and

$$
\gamma_{\mu, r}\left(n \omega_{\mu}\right)=\gamma_{\mu, r}(0)+n \mathfrak{w}_{m, r} \vec{k}, \quad n \in \mathbb{Z}
$$

$$
\gamma_{\mu, r}\left(\frac{\omega_{\mu}}{2}+n \omega_{m u}\right)=\gamma_{\mu, r}\left(\frac{\omega_{\mu}}{2}\right)+n \mathfrak{w}_{m, r} \vec{k}, \quad n \in \mathbb{Z}
$$

All the other points are regular. This shows that pseudospherical helicoids of electric type are of translational kind and have parity $\epsilon=1$. The profile reaches the maximal distance from the screw axis at the points

$$
\gamma_{\mu}\left(n \omega_{\mu}\right)=\gamma_{\mu, r}(0)+n \mathfrak{w}_{\mu, r} \vec{k} .
$$

The outer radius is then

$$
\mathfrak{r}_{\mu, r}^{+}=\frac{\sqrt{m} \cosh (r)}{m+\sinh ^{2}(r)} .
$$


This implies that the aspect ratid ${ }^{5}$ is

$$
\begin{aligned}
\mathfrak{d}_{\mu, r}= & \frac{2 \sqrt{m+\sinh ^{2}(r)}}{m \cosh (r)}\left(\left(m+\cosh ^{2}(r)-1\right) K\left(\frac{1}{m}\right)-m E\left(\frac{1}{m}\right)\right. \\
& \left.-\cosh ^{2}(r) \Pi\left(-\operatorname{csch}^{2}(r), \frac{1}{m}\right)\right),
\end{aligned}
$$

which proves the claim.
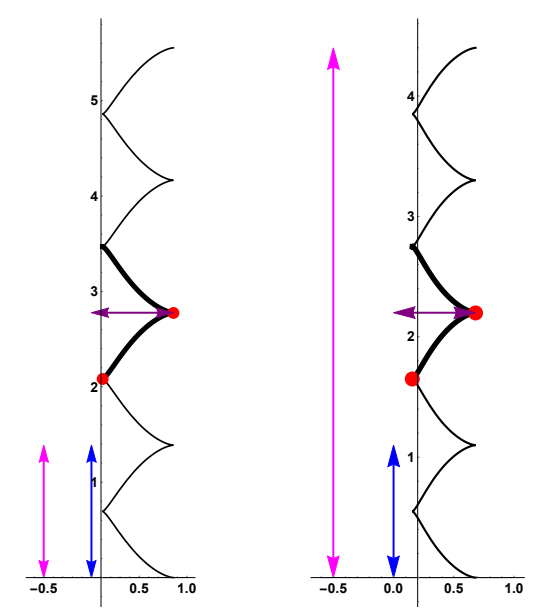

Figure 5. The planar profiles of two twisted columns of electric type with wave numbers 1 and 4, respectively.

4.2. Existence of pseudospherical twisted columns with prescribed invariants. We start by giving the following.

Definition 6. Let

$$
\mathcal{M}_{-}=\mathbb{R}^{+} \times(0,1), \quad \mathcal{M}_{+}=\mathbb{R}^{+} \times(1,+\infty) .
$$

For a positive integer $n \in \mathbb{N}$ and a positive real number $d \in \mathbb{R}^{+}$, let

$$
\begin{aligned}
\mathcal{C}_{n}^{ \pm} & =\left\{(r, m) \in \mathcal{M}_{ \pm} \mid \mathfrak{p}_{\mu, r}=n \mathfrak{w}_{\mu, r}\right\}, \\
\mathcal{D}_{d}^{ \pm} & =\left\{(r, m) \in \mathcal{M}_{ \pm} \mid \mathfrak{d}_{\mu, r}=d\right\},
\end{aligned}
$$

where, depending on whether $\mathcal{C}_{n}^{-}, \mathcal{D}_{d}^{-}$or $\mathcal{C}_{n}^{+}, \mathcal{D}_{d}^{+}$are considered, $\mathfrak{p}_{\mu, r}, \mathfrak{w}_{\mu, r}$ and $\mathfrak{d}_{\mu, r}$ are as in Lemma 8 or Lemma 9 above. Accordingly, we call $\mathcal{C}_{n}^{-}, \mathcal{D}_{d}^{-}$and $\mathcal{C}_{n}^{+}$, $\mathcal{D}_{d}^{+}$the modular curves of magnetic type and of electric type, respectively.

Remark 8. From Lemmas 8 and 9 , it follows that a pseudospherical helicoid with parity $\epsilon=-1$ (resp., $\epsilon=1$ ) is a twisted column if and only if $(r, m) \in \mathcal{C}_{n}^{-}$(resp., $(r, m) \in \mathcal{C}_{n}^{+}$), where $n \in \mathbb{N}$ is its wave number ${ }^{6}$ Consequently, a pseudospherical helicoid with parameters $(\mu, r)$ is a twisted column with parity $\epsilon=-1$ (resp., $\epsilon=1$ ), wave number $\mathfrak{n}$, and aspect ratio $\mathfrak{d}$ if and only if $(r, m) \in \mathcal{C}_{\mathfrak{n}}^{-} \cap \mathcal{D}_{\mathfrak{d}}^{-}$(resp., $\left.(r, m) \in \mathcal{C}_{\mathfrak{n}}^{+} \cap \mathcal{D}_{\mathfrak{d}}^{+}\right)$. 

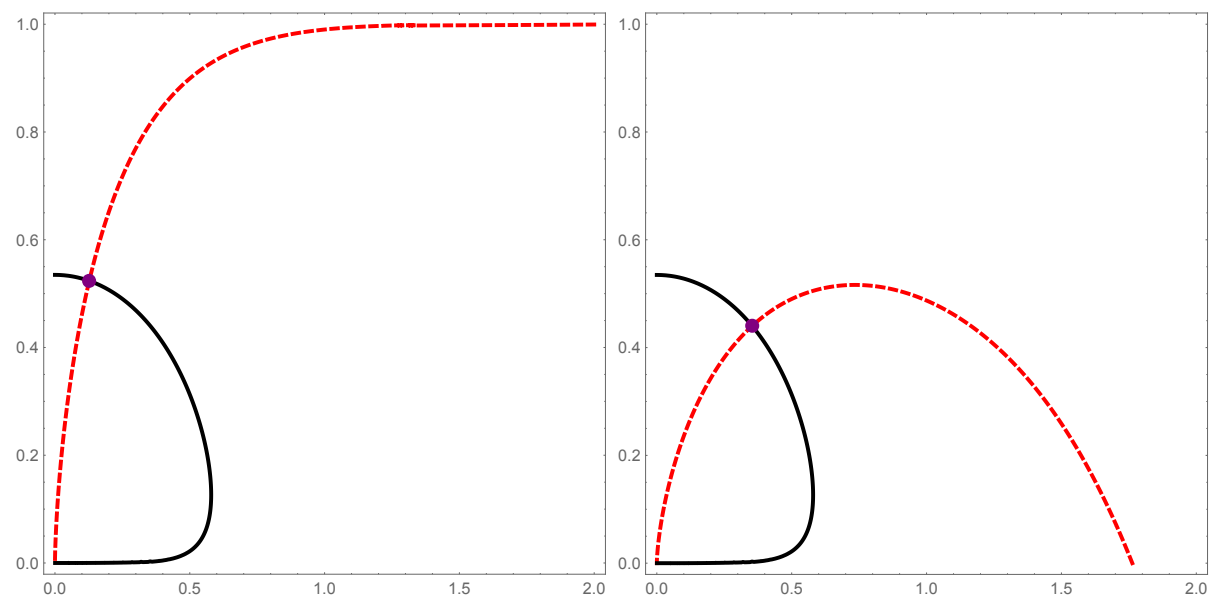

Figure 6 . The modular curves $\mathcal{C}_{n}^{-}$(dashed lines) and $\mathcal{D}_{d}^{-}$(solid lines) for $n=1$ (left), $n=3$ (right) and $d$ equal to the golden ratio. The parameters of the corresponding twisted columns are $\mu \approx 1.90951, \mu \approx 2.27181$ and $r=0.127237, r=0.353348$, respectively.

Lemma 10. For given $n \in \mathbb{N}$ and $d>0$, there exists a pseudospherical twisted column of magnetic type with wave number $n$ and aspect ratio $d$.

Proof. It suffices to prove that $\mathcal{C}_{n}^{-} \cap \mathcal{D}_{d}^{-}$consists of a single point. From the previous Lemmas it follows that the points of $\mathcal{C}_{n}^{-} \cap \mathcal{D}_{d}^{-}$are the solutions of the system

$$
\begin{aligned}
& \cosh ^{2}(r) \Pi\left(-\sinh ^{2}(r), m\right)=E(m)+\frac{\pi \cosh (r) \sinh (r)}{n \sqrt{m+\sinh ^{2}(r)}}, \\
& \cosh ^{2}(r) \Pi\left(-\sinh ^{2}(r), m\right)=E(m)+d \frac{\sqrt{m(1-m)}}{2 \sqrt{m+\sinh ^{2}(r)}} .
\end{aligned}
$$

This implies that $r=\varrho_{n, d}(m)$, where $\varrho_{n, d}:(0,1) \rightarrow \mathbb{R}$ is defined by

$$
\varrho_{n, d}(y)=\frac{1}{2} \operatorname{arcsinh}\left(\frac{d \cdot n \sqrt{y(1-y)}}{\pi}\right) .
$$

Substituting 4.4 into the second equation of 4.3), we find that $m \in(0,1)$ is a zero of the function

$$
\begin{gathered}
h_{n, d}(y)=-2 E(y)+2 \cosh ^{2}\left(\varrho_{n, d}(y)\right) \Pi\left(-\sinh ^{2}\left(\varrho_{n, d}(y)\right), y\right) \\
-d \sqrt{\frac{y(1-y)}{y+\sinh ^{2}\left(\varrho_{n, d}(y)\right)}},
\end{gathered}
$$

\footnotetext{
${ }^{5}$ Since $\epsilon=1$, the aspect ratio is $\mathfrak{w} / \mathfrak{r}^{+}$.

${ }^{6}$ Recall that in Lemmas 8 and $9, m$ stands for $\mu^{-1}$.
} 


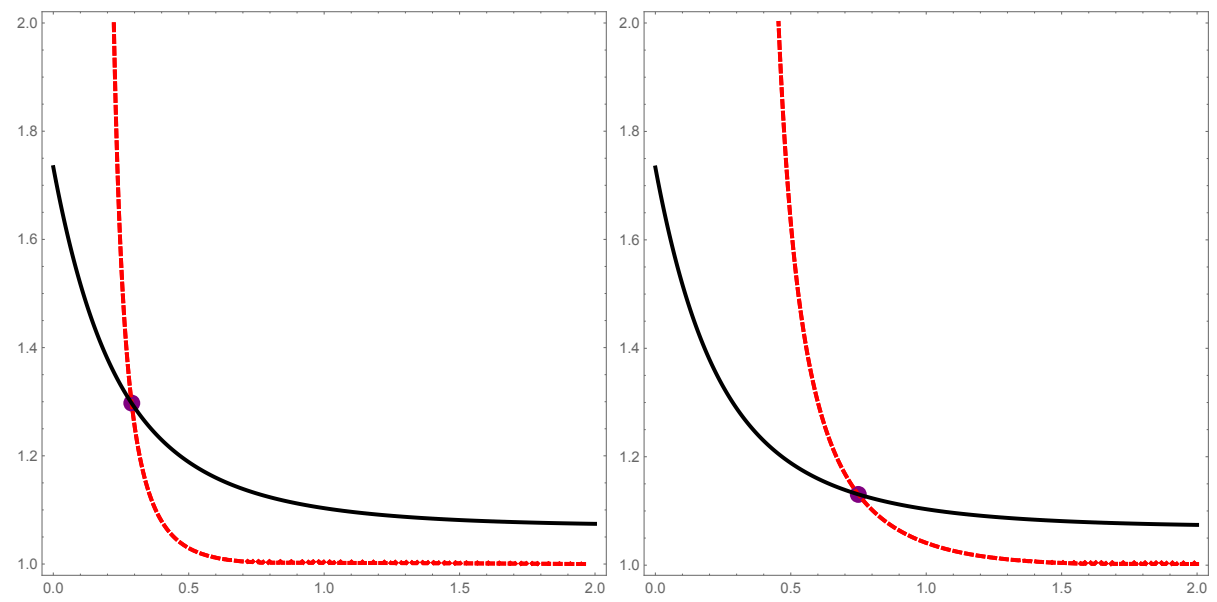

Figure 7 . The modular curves $\mathcal{C}_{n}^{+}$(dashed lines) and $\mathcal{D}_{d}^{+}$(solid lines) for $n=1$ (left), $n=3$ (right) and $d$ equal to the golden ratio. The parameters of the corresponding twisted columns are $\mu \approx 0.7708, \mu \approx 0,8844$ and $r=0.289, r=0.749$, respectively.

which is defined on $(0,1)$. The latter is a strictly increasing real-analytic function, such that

$$
\lim _{y \rightarrow 1^{-}} h_{n, d}(y)=+\infty, \quad \lim _{y \rightarrow 0^{+}} h_{n, d}(y)=-\frac{2 d \pi}{\sqrt{n^{2} d^{2}+4 \pi^{2}}}<0 .
$$

Therefore, for given $n \in \mathbb{N}$ and $d>0$, there exists a unique $m_{n, d} \in(0,1)$, such that $h_{n, d}\left(m_{n, d}\right)=0$. We have thus proved that

$$
\mathcal{C}_{n}^{-} \cap \mathcal{D}_{d}^{-}=\left(\varrho_{n, d}\left(m_{n, d}\right), m_{n, d}\right),
$$

which yields the required result.

Lemma 11. For given $n \in \mathbb{N}$ and $d>0$, there exists a pseudospherical twisted column of electric type with wave number $n$ and aspect ratio $d$.

Proof. We argue as in Lemma 10. The points of $\mathcal{C}_{n}^{+} \cap \mathcal{D}_{d}^{+}$are the solutions of the system

$$
\begin{aligned}
& \frac{\pi \sqrt{m} \sinh (2 r)}{\sqrt{m+\sinh ^{2}(r)}}+2 n\left[m E\left(\frac{1}{m}\right)-\left(\cosh ^{2}(r)+m-1\right) K\left(\frac{1}{m}\right)\right. \\
& \left.\quad+\cosh ^{2}(r) \Pi\left(-\operatorname{csch}^{2}(r), \frac{1}{m}\right)\right]=0, \\
& \frac{d m \cosh (r)}{\sqrt{m+\sinh ^{2}(r)}}+2\left[m E\left(\frac{1}{m}\right)-\left(\cosh ^{2}(r)+m-1\right) K\left(\frac{1}{m}\right)\right. \\
& \left.\quad+\cosh ^{2}(r) \Pi\left(-\operatorname{csch}^{2}(r), \frac{1}{m}\right)\right]=0 .
\end{aligned}
$$



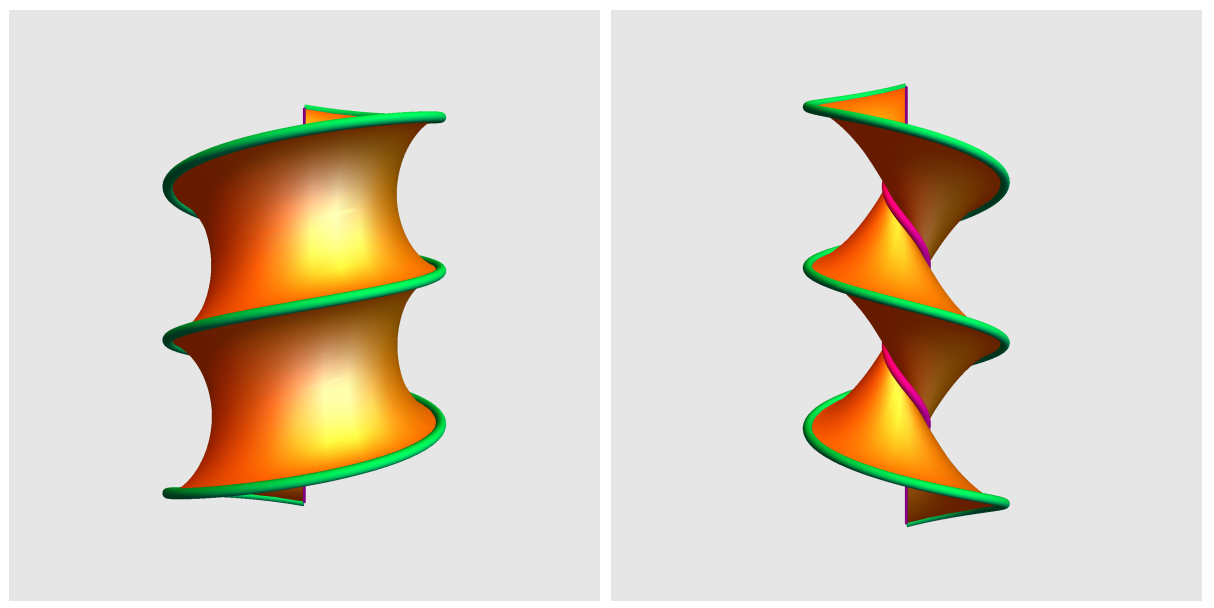

Figure 8. Magnetic (left) and electric (right) pseudospherical twisted columns with $\mathfrak{n}=1$ and $\mathfrak{d}=\phi, \phi=$ golden ratio.

The first equation implies that $\Pi\left(-\operatorname{csch}^{2}(r), 1 / m\right)$ is equal to

$$
\begin{aligned}
& \frac{n \operatorname{sech}^{2}(r)(\cosh (2 r)+2 m-1)\left(-2 m E\left(\frac{1}{m}\right)+(\cosh (2 r)+2 m-1) K\left(\frac{1}{m}\right)\right)}{4 n\left(m+\sinh ^{2}(r)\right)} \\
& -\frac{\pi \sqrt{m\left(m+\sinh ^{2}(r)\right)} \tanh (r)}{n\left(m+\sinh ^{2}(r)\right)} .
\end{aligned}
$$

Substituting this expression in the second equation of 4.5), we deduce that $r=$ $\varrho_{n, d}(m)$, where $\varrho_{n, d}: \mathbb{R}^{+} \rightarrow \mathbb{R}$ is defined by

$$
\varrho_{n, d}(y)=\operatorname{arcsinh}\left(\frac{n \sqrt{y} d}{2 \pi}\right) .
$$

Substituting (4.6) into the first equation of 4.5), we find that $m \in(1,+\infty)$ is a zero of the function

$$
\begin{aligned}
h_{n, d}(y)= & \frac{2 d \pi^{2} \sqrt{d^{2} n^{2} y^{2}+4 \pi^{2} y}}{\sqrt{d^{2} n^{2}+4 \pi^{2}}}+\left(4 y \pi^{2} E\left(\frac{1}{y}\right)-y\left(d^{2} n^{2}+4 \pi^{2}\right) K\left(\frac{1}{y}\right)\right. \\
& \left.+\left(d^{2} n^{2} y+4 \pi^{2}\right) \Pi\left(-\frac{4 \pi^{2}}{d^{2} n^{2} y}, \frac{1}{y}\right)\right),
\end{aligned}
$$

which is defined on $(0,+\infty)$. This is a strictly increasing real-analytic function such that

$$
\lim _{y \rightarrow 0^{+}} h_{n, d}(y)=-\infty, \quad \lim _{y \rightarrow+\infty} h_{n, d}(y)=+\infty .
$$

Therefore, for given $n \in \mathbb{N}$ and $d>0$, there exists a unique $m_{n, d} \in(1,+\infty)$ such that $h_{n, d}\left(m_{n, d}\right)=0$. We have thus proved that

$$
\mathcal{C}_{n}^{+} \cap \mathcal{D}_{d}^{+}=\left(\varrho_{n, d}\left(m_{n, d}\right), m_{n, d}\right),
$$

which yields the required result. 
Remark 9 (Number of singular helices). In the magnetic-type case, the wave number tells how many singular helices are contained in the pseudospherical helicoid. In the electric-type case, instead, the number of singular helices in the pseudospherical helicoid is two times the wave number (see Example 1). This result should be compared with the local analysis of Brander [2] about the pseudospherical fronts containing circular helices as singular points.

4.3. End of the proof. To conclude the proof of Theorem 2 we are left with the following.

Lemma 12. Let $S$ and $S^{\prime}$ be two right-handed (or left-handed) pseudospherical twisted columns with invariants $(\epsilon, \mathfrak{n}, \mathfrak{d})$ and $\left(\epsilon^{\prime}, \mathfrak{n}^{\prime}, \mathfrak{d}^{\prime}\right)$. If $(\epsilon, \mathfrak{n}, \mathfrak{d}) \neq\left(\epsilon^{\prime}, \mathfrak{n}^{\prime}, \mathfrak{d}^{\prime}\right)$ then $S$ and $S^{\prime}$ cannot be congruent to each other.

Proof. First, we show that if $\epsilon \neq \epsilon^{\prime}$, then $S$ and $S^{\prime}$ cannot be congruent to each other. Suppose, on the contrary, that $S$ and $S^{\prime}$ are congruent. By possibly acting with a rigid motion, we may assume $S=S^{\prime}$. Thus, if $\Gamma_{*}$ is a fundamental domain of the planar profile of $S$, then $\Gamma_{*}$ must be a fundamental domain of the planar profile of $S^{\prime}$. On the other hand, if $\epsilon \neq \epsilon^{\prime}$, the number of cusps of the fundamental domains are different, which is a contradiction. Next, suppose $\epsilon=\epsilon^{\prime}$. If $\mathfrak{n} \neq \mathfrak{n}^{\prime}$ and $\epsilon=\epsilon^{\prime}$, then the number of connected components of the singular loci of $S$ and $S^{\prime}$ are different. Thus $S$ and $S^{\prime}$ cannot be congruent. We now prove that if $\epsilon=\epsilon^{\prime}$ and $\mathfrak{n}=\mathfrak{n}^{\prime}$, but $\mathfrak{d} \neq \mathfrak{d}^{\prime}$, then $S$ and $S^{\prime}$ are not congruent. Suppose not, then they must have the same pitch and the same inner and outer radii. If $\mathfrak{p}=\mathfrak{p}^{\prime}$ and $\mathfrak{n}=\mathfrak{n}^{\prime}$, then $\mathfrak{w}=\mathfrak{w}^{\prime}$. Since they have the same wave numbers, parities, inner and outer radii, they must have also the same aspect ratios, which is a contradiction.

Example 1. We now examine the pseudospherical twisted columns of the goldenmean series (that is, $\mathfrak{d}$ is equal to the golden ratio). The numerical evaluation of the parameters $(\mu, r)$ and the visualization have been done with the software Mathematica. The following is the table of the approximate values of the parameters $(\mu, r)$ of the twisted columns (magnetic type) with phenomenological invariants $(\epsilon, \mathfrak{n}, \mathfrak{d})=(-1, n, \phi), n=1, \ldots, 6$,

\begin{tabular}{|c|c|c|c|c|c|c|}
\hline$n$ & 1 & 2 & 3 & 4 & 5 & 6 \\
$\mu$ & 1.90951 & 2.03576 & 2.27181 & 2.65802 & 3.25281 & 4.13094 \\
$r$ & 0.127237 & 0.247275 & 0.353348 & 0.439982 & 0.504218 & 0.546398 \\
\hline
\end{tabular}

The following is the table of the approximate values of the parameters $(\mu, r)$ of the twisted columns (electric type) with phenomenological invariants $(\epsilon, \mathfrak{n}, \mathfrak{d})=$ $(1, n, \phi), n=1, \ldots, 6$,

\begin{tabular}{|c|c|c|c|c|c|c|}
\hline$n$ & 1 & 2 & 3 & 4 & 5 & 6 \\
$\mu$ & 0.770862 & 0.849115 & 0.884453 & 0.902344 & 0.912336 & 0.918378 \\
$r$ & 0.289255 & 0.533287 & 0.749346 & 0.939799 & 1.1074 & 1.25549 \\
\hline
\end{tabular}

Figure 8 reproduces pseudospherical twisted columns with invariants $(-1,1, \phi)$ and $(1,1, \phi)$, respectively. The magnetic-type one has one singular helix while the electric-type one has two singular helices. Figure 9 reproduces pseudospherical twisted columns with invariants $(-1,2, \phi)$ and $(1,2, \phi)$, respectively. The magnetictype one has two singular helices while the electric-type one has four singular helices. Figure 10 depicts pseudospherical twisted columns with invariants $(-1,3, \phi)$ and $(1,3, \phi)$, respectively. The magnetic-type one has three singular helices, while the 

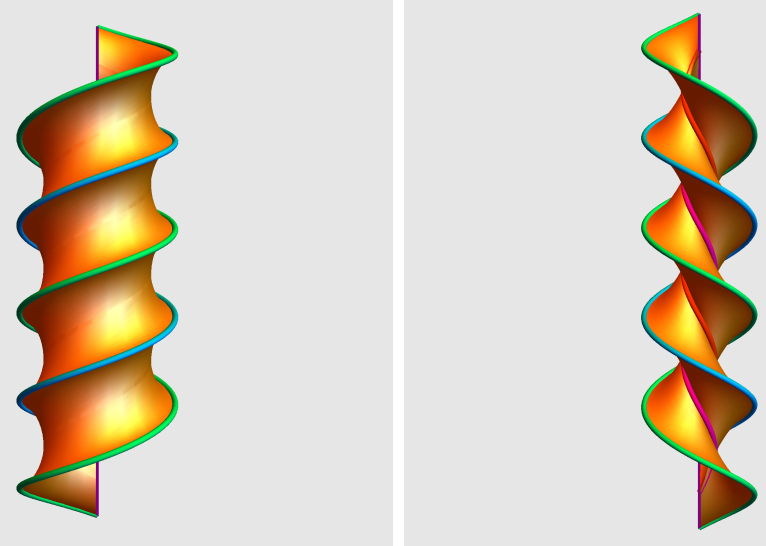

Figure 9. Magnetic (left) and electric (right) pseudospherical twisted columns with $\mathfrak{n}=2$ and $\mathfrak{d}=\phi, \phi=$ golden ratio.
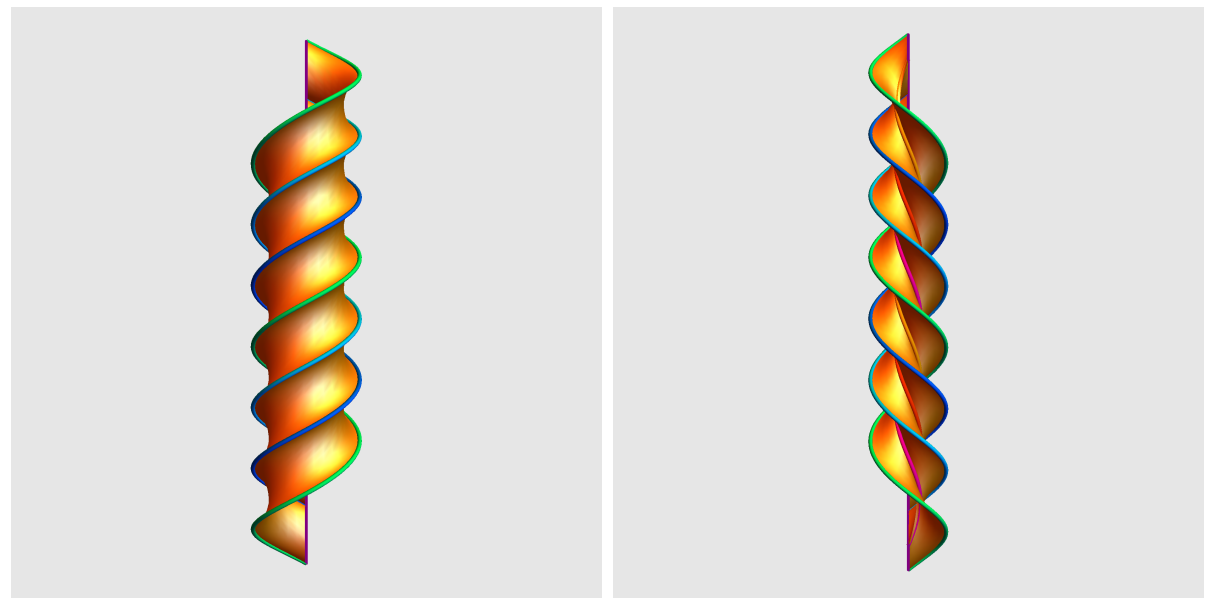

Figure 10. Magnetic (left) and electric (right) pseudospherical twisted columns with $\mathfrak{n}=3$ and $\mathfrak{d}=\phi, \phi=$ golden ratio.

electric-type one has six singular helices. For the magnetic-type twisted columns we have chosen the right-handed orientation, while for the electric-type ones we have chosen the left-handed orientation.

\section{REFERENCES}

[1] L. Bianchi, Lezioni di geometria diferenziale, terza edizione, Zanichelli, Bologna, 1927.

[2] D. Brander, Pseudospherical surfaces with singularities, Ann. Mat. Pura Appl. (4) (2016), DOI: $10.1007 / \mathrm{s} 10231-016-0601-8$; arXiv:1502.04876 [math.DG].

[3] M. Brück, X. Du, J. Park, and C.-L. Terng, The submanifold geometries associated to Grassmannian systems, Mem. Amer. Math. Soc. 155 (2002), no. 735, viii+95 pp.

[4] F. E. Burstall and M. Kilian, Equivariant harmonic cylinders, Q. J. Math. 57 (2006), no. $4,449-468$. 
[5] A. Calini and T. Ivey, Bäcklund transformations and knots of constant torsion, J. Knot Theory Ramifications 7 (1998), no. 6, 719-746.

[6] M. P. Do CARmo AND M. DAJCZER, Helicoidal surfaces with constant mean curvature, Tohoku Math. J. (2) 34 (1982), no. 3, 425-435.

[7] A. Dzhalilov, E. Musso, And L. Nicolodi, Conformal geometry of timelike curves in the (1+2)-Einstein universe, Nonlinear Anal. 143 (2016), 224-255.

[8] L. P. Eisenhart, A treatise on the differential geometry of curves and surfaces, Dover Publications, Inc., New York, 1960.

[9] M. Fels and P. Olver, Moving coframes. I. A practical algorithm, Acta Appl. Math. 51 (1998), no. 2, 161-213.

[10] M. Fels and P. Olver, Moving coframes. II. Regularization and theoretical foundations, Acta Appl. Math. 55 (1999), no. 2, 127-208.

[11] G. Fischer, Mathematical models: Photograph volume and commentary, Vieweg, Braunschweig and Wiesbaden, 1986.

[12] D. Hilbert, Grundlagen der Geometrie, B. G. Teubner, Stuttgart, 1999.

[13] G. R. Jensen, E. Musso, and L. Nicolodi, Surfaces in classical geometries. A treatment by moving frames, Universitext, Springer, Cham, 2016.

[14] D. F. Lawden, Elliptic functions and applications, Series in Applied Mathematical Science, 80, Springer-Verlag, New York, 1989.

[15] R. MCLachlan, A gallery of constant-negative-curvature surfaces, Math. Intelligencer 16 (1994), no. 4, 31-37.

[16] F. Minding, Wie sich entscheiden lässt, ob zwei gegebene krumme Flächen auf einander abwickelbar sind oder nicht; nebst Bemerkungen über die Flächen von unveränderlichem Krümmungsmaasse, J. Reine Angew. Math. 19 (1839), 370-387.

[17] E. Musso And L. Nicolodi, Tableaux over Lie algebras, integrable systems, and classical surface theory, Comm. Anal. Geom. 14 (2006), no. 3, 475-496.

[18] E. Musso And L. Nicolodi, A class of overdetermined systems defined by tableaux: Involutiveness and Cauchy problem, Phys. D 229 (2007), no. 1, 35-42.

[19] E. Musso AND L. NicOlOdi, Closed trajectories of a particle model on null curves in anti-de Sitter 3-space, Classical Quantum Gravity 24 (2007), no. 1, 5401-5411.

[20] E. Musso And L. Nicolodi, Reduction for constrained variational problems on 3D null curves, SIAM J. Control Optim. 47 (2008), no. 3, 1399-1414.

[21] E. Musso And L. Nicolodi, Hamiltonian flows on null curves, Nonlinearity 23 (2010), $2117-2129$

[22] E. Musso ANd L. Nicolodi, Holomorphic differentials and Laguerre deformation of surfaces, Math. Z. 284 (2016), no. 3-4, 1089-1110.

[23] E. Musso And L. Nicolodi, Quantization of the conformal arclength functional on space curves, Comm. Anal. Geom. 25 (2017), no. 1 (to appear); arXiv:1501.04101 [math.DG].

[24] P. Olver, Applications of Lie groups to differential equations, Graduate Texts in Mathematics, 107, Springer-Verlag, New York, 1986.

[25] A. Ovchinninkov, Gallery of pseudospherical surfaces, Nonlinearity \& geometry (Warsaw, 1995), 41-60, PWN, Warsaw, 1998.

[26] O. Perdomo, A dynamical interpretation of the profile curve of CMC twizzler surfaces, Pacific J. Math. 258 (2012), no. 2, 459-485.

[27] R. S. Palais and C.-L. Terng, Critical Point Theory and Submanifold Geometry, Lecture Notes in Mathematics, 1353, Springer-Verlag, Berlin, 1988.

[28] R. S. Palais, The symmetries of solitons, Bull. Amer. Math. Soc. (N.S.) 34 (1997), 339-403.

[29] A. G. Popov and E. V. Maevskil, Analytical approaches to the investigation of the sineGordon equation and pseudospherical surfaces, J. Math. Sci. (N.Y.) 142 (2007), no. 5, 2377-2418.

[30] A. Popov, Lobachevsky geometry and modern nonlinear problems, Translated from the 2012 Russian original by Andrei Iacob, Birkhäuser/Springer, Cham, 2014.

[31] E. G. Poznyak, Geometric interpretation of regular solutions of the equation $z_{x y}=\sin z$ (Russian), Differentsial'nye Uravneniya 15 (1979), no. 7, 1332-1336.

[32] C. Rogers AND W. K. Schief, Bäcklund and Darboux transformation, geometry and modern applications in soliton theory, Cambridge Texts in Applied Mathematics, Cambridge University Press, Cambridge, 2002. 
[33] C. Rogers And W. K. Schief, Modulated waves and helicoidal pseudospherical surfaces in nonlinear inhomogeneous elasticity, J. Phys. A 43 (2010), no. 10, 105206, 12 pp.

[34] C. Rogers, W. K. Schief, And J. Wylie, Wave propagation in ideally hard inhomogeneous elastic materials associated with pseudospherical surfaces, Internat. J. Engrg. Sci. 41 (2003), no. $17,1965-1974$.

[35] C. Rogers, W. K. Schief, and A. Szereszewski, Loop soliton interaction in an integrable nonlinear telegraphy model: reciprocal and Bäcklund transformations, J. Phys. A $\mathbf{4 3}$ (2010), no. $38,385210,16$ pp.

[36] I. M. Roussos, A geometric characterization of helicoidal surfaces of constant mean curvature, Publ. Inst. Math. (Beograd) (N.S.) 43 (57) (1988), 137-142.

[37] R. SASAKI, Soliton equations and pseudospherical surfaces, Nuclear Phys. B 154 (1979), no. $2,343-357$.

[38] K. Tenenblat, Transformations of manifolds and applications to differential equations, Pitman Monographs and Surveys in Pure and Applied Mathematics, 93, Longman, Harlow, 1998.

[39] C.-L. Terng and K. Uhlenbeck, Geometry of solitons, Notices Amer. Math. Soc. 47 (2000), no. $1,17-25$.

(E. Musso) Dipartimento di Scienze Matematiche, Politecnico di Torino, Corso Duca Degli Abruzzi 24, I-10129 Torino, Italy

E-mail address: emilio.musso@polito.it

(L. Nicolodi) Dipartimento di Scienze Matematiche, Fisiche e Informatiche, UniverSità di Parma, Parco Area delle Scienze 53/A, I-43124 Parma, Italy

E-mail address: lorenzo.nicolodi@unipr.it 